\title{
A Simple Encompassing Test for the Deterministic and Bilinear Unit Root Models
}

\author{
Wojciech W. Charemza ${ }^{1}$ and Svetlana Makarova ${ }^{2}$ \\ ${ }^{1}$ National Bank of Poland and University of Leicester, UK \\ ${ }^{2}$ European University at St. Petersburg, Russia and National Bank of \\ Poland
}

\begin{abstract}
A new parameters' encompassing test is proposed for deciding between the deterministic unit root processes with a structural break and the bilinear unit root model without such break. The test consists in testing three sets of hypotheses regarding parameters in a simple regression model. The test uses the $t$-ratio and $F$-statistics, of non-trivial distributions under the null hypothesis. The finite sample distributions for the relevant statistics are tabulated and the asymptotic distribution of the $F$-test is derived. The test has been applied for the daily stock price indices for 66 countries, for the period 1992-2001. The results support the conjecture that the bilinear model dominates the structural break model more often than the other way around. Also, it is likely that in practical applications the bilinear unit root process might be mistaken for the deterministic unit root process with a structural break.
\end{abstract}

Financial support of INTAS project No. 03-51-3714 Nonstationary multivariate and nonlinear econometric models: theory and applications is gratefully acknowledged.

\section{The Model}

The paper considers two particular stochastic processes embedded within the following general process:

$$
u_{t}=d_{t}+y_{t}, \quad y_{t}=\rho_{t} y_{t-1}+v_{t}
$$

where $d_{t}$ is a deterministic part, $E\left(y_{0}^{p}\right)^{2}<\infty, v_{t}=\delta(L) \varepsilon_{t}=\sum_{j=0}^{\infty} \delta_{j} \varepsilon_{t-j}$ and $\sum_{j=0}^{\infty} j\left|\delta_{j}\right|<\infty$ (see Ng and Perron, 2001), $\rho_{t}$ is series of (possibly degenerated) random variables, $\varepsilon_{t} \sim \operatorname{IID}\left(0, \sigma^{2}\right)$ and $t=1,2, \ldots, T$. The deterministic part in (1) is given as:

$$
d_{t}=\text { const. }+\gamma B_{t}
$$

where $B_{t}$ is a variable indicating a nonlinear change in the deterministic part of the process. The most commonly used form of the break is either the 'slope':

$$
B_{t}=\left\{\begin{array}{cl}
\left(t-T_{B}\right), & \text { for } t>T_{B} \\
0, & \text { otherwise }
\end{array}\right.
$$

or the 'step':

$$
B_{t}=\left\{\begin{array}{l}
0, \text { for } t<T_{B} \\
1, \text { otherwise }
\end{array}\right.
$$


and $T_{B}$ indicates the position of the beginning of the structural break in the series. A number of other, more complex, specifications of $B_{t}$ is also possible (see Vogelsang and Perron, 1998; Harvey and Mills, 2002).

As far as the unit root part in (1) is concerned, the following terminology is introduced:

(a) The unit root process has a deterministic unit root and is linear if in (1) $\rho_{t}=1$ and in (2) $\gamma=0$.

(b) The unit root process is deterministic unit root and is nonlinear if $\rho_{t}=1$ and $\gamma \neq 0$.

(c) The unit root process has a stochastic unit root if $\rho_{t}$ is a non-degenerated random variable with the expected value of one. In this paper we only consider the stochastic unit root process without a deterministic part, that is where $\gamma=0$.

The first two processes, (a) and (b), constitute a family of the deterministic unit root $(U R D)$ processes. The literature on testing and evaluation of the URD processes is well developed. In particular, the nonlinear $U R D$ models (and such models are considered in this paper) are analysed in Kim et al. (2000); Ng and Perron (2001); Perron (1989, 1990, 1997); Vogelsang and Perron (1998); Zivot and Andrews (1992). Among the stochastic unit root processes, this paper focuses on the bilinear unit root (URB) process of the first order (see Charemza et al., 2005), defined as:

$$
\rho_{t}=1+b \varepsilon_{t-1}, \quad b \neq 0, \text { where } \operatorname{cov}\left(\rho_{t}, \varepsilon_{t}\right)=0 .
$$

For other specifications of the stochastic unit root models see e.g. Granger and Swanson (1997); Leybourne et al. (2000); McCabe and Tremayne (1995); Sollis et al. (2000). We consider a simple case of (1) where $d_{t}=0$ and $\nu_{t}=\varepsilon_{t}$, which gives:

$$
y_{t}=\left(1+b \varepsilon_{t-1}\right) y_{t-1}+\varepsilon_{t}
$$

For $b=0,(5)$ becomes a simple $U R D$ process, without any deterministic part (a random walk). The process (5) is nonstationary, since $1+b^{2} \sigma^{2} \geq 1$ (see Granger and Andersen, 1978). In Charemza et al. (2005) it is shown that, under the null hypothesis that $b=0$ the Student $t$ statistic for $\hat{b}$ in the regression equation:

$$
\Delta y_{t}=\hat{b} y_{t-1} \Delta_{t-1}+e_{t}
$$

where $e_{t}$ are the regression residuals, has an asymptotic standard normal distribution. It is also shown that the similar test statistics can be formulated for a regression containing an intercept and for demeaned series of first differences of $y_{t}$. If the relationship between $\nu_{t}$ and $\varepsilon_{t}$ is more complex, that is, where $\nu_{t}$ is described by a fully defined moving average process, it is possible to add augmentations to (6), as in the Augmented Dickey-Fuller test.

\section{The Test}

In order to decide whether a $U R D$ (possibly nonlinear, with a deterministic break) or a $U R B$ (without a deterministic break) model describes the series in a more accurate way, 
a simple encompassing exercise has been performed. It is based on the following regression:

$$
\Delta y_{t}=\hat{c}+\hat{b} y_{t-1} \Delta y_{t-1}+\hat{\gamma} B_{t}+\sum_{i=1}^{k} c_{i} \Delta y_{t-i}+e_{t}
$$

so that $\hat{c}, \hat{b}$ and $\hat{\gamma}$ are the estimates for $c, b$ and $\gamma$. There are three sets of the hypotheses of interest to be considered:

(A) $H_{0}: b=0$ and $\gamma=0$ and $H_{1}: b \neq 0$ and/or $\gamma \neq 0$

(B) $H_{0}: \gamma=0$ and $H_{1}: \gamma \neq 0$

(C) $H_{0}: b=0$ and $H_{1}: b \neq 0$

The rejection of $H_{0}$ in (A) confirms that the unit root process is either subject to bilinearity, or a structural break. Further on, the rejection of $H_{0}$ in (B) confirms the existence of the deterministic structural break without much evidence of the $U R B$ process. If, however, $H_{0}$ is rejected in (A) and (C) and is not rejected in (B), this would implicitly confirm the existence of the $U R B$ and not the $U R D$ with break process.

For testing these sets of hypotheses we use Student $t$-statistics and, additionally in (A), the $F$ statistic for testing the joint significance of $b$ and $\gamma$ on the basis of their estimators $\hat{b}$ and $\hat{\gamma}$. In (A), under the null hypothesis, the asymptotic distribution of the $t$-statistic for $\hat{\gamma}$, denoted as $t(\hat{\gamma})$ is standard normal. However, since the finite sample distribution of $t(\hat{b})$ is non-standard, the distribution of the $F$-statistic is unknown and has to be approximated numerically. The limit distribution of $F$-statistic in case of 'step' is given by the following theorem:

Theorem. Let the series $y_{t}$ be generated by

$$
y_{t}=y_{t-1}+\varepsilon_{t}, \varepsilon_{t} \sim \operatorname{IID}\left(0, \sigma^{2}\right), y_{0}=0, t=1,2, \ldots, T .
$$

Consider the regression model (7) with $B_{t}$ defined by (4). Denote by $\lambda$ the ratio of time of break $T_{B}$ to the sample size $T$ and assume that it is constant (as $T$ goes to infinity), that is

$$
\frac{T_{B}}{T}=\lambda=\text { cons }
$$

Under the null hypothesis of $b=\gamma=0$, as $T \rightarrow \infty$ the F-statistic has a limit distribution of the form:

$$
\begin{array}{r}
F \Rightarrow \frac{1}{2}\left[\begin{array}{c}
\left(\int_{0}^{1} W_{1}(t) d W_{2}(t)\right)^{2} \\
\int_{0}^{1} W_{1}^{2}(t) d t
\end{array}+\frac{(1-\sqrt{\lambda})(4-\lambda)}{\lambda(1+\sqrt{\lambda})} W_{1}^{2}(1)\right] \sim \\
\frac{1}{2}\left[\chi_{1}^{2}(1)+\frac{(1-\sqrt{\lambda})(4-\lambda)}{\lambda(1+\sqrt{\lambda})} \chi_{2}^{2}(1)\right],
\end{array}
$$

where $\Rightarrow$ denotes weak convergence when $T \rightarrow \infty, W_{1}, W_{2}$ are two independent Wiener processes on $[0,1]$, and $\chi_{1}^{2}(1), \chi_{2}^{2}(1)$, are two independent chi-squared distributions with one degree of freedom ${ }^{3}$.

Proof of the Theorem is based on the following Lemma:

\footnotetext{
${ }^{3}$ We are grateful to Mikhail Lifshits for additional comment on the independence of the chi squared variables in this theorem.
} 
Lemma. Let the series $y_{t}$ be generated by (8). For the regression model (7) with BREAK $_{t}$ defined by (4) under the null hypothesis of $b=\gamma=0$, as $T \rightarrow \infty$ :

1) $\sqrt{T} \hat{c} \Rightarrow \frac{\sigma}{\sqrt{\lambda}} W_{1}(1)$

2) $T \hat{b} \Rightarrow \frac{\int_{0}^{1} W_{1}(t) d W_{2}(t)}{\sigma \int_{0}^{1} W_{1}^{2}(t)}$,

3) $\sqrt{T} \hat{\gamma} \Rightarrow \frac{\sigma}{\sqrt{\lambda}(1+\sqrt{\lambda})} W_{1}(1)$,

where $\Rightarrow$ denotes weak convergence, $W_{1}, W_{2}$ are two independent Wiener processes on $[0,1]$ and $\lambda$ is defined by (9).

The proofs of Lemma and the Theorem above are given in Appendix A.

It can also be shown that in (B), under the null hypothesis, the asymptotic distribution of $t(\hat{\gamma})$ is not normal and it depends on $b$. Moreover for $t(\hat{b})$ in $(\mathrm{C})$, under the null hypothesis, that is for the Student- $t$ statistic for the parameter $b$, the asymptotic normality is no longer the case, although the limit distribution remains well-defined as long as $0<b \leq 1 / \sqrt{T}$ (see Lifshits, 2002).

In order to facilitate testing, critical values for particular tests (assuming additionally the normality of $\varepsilon_{t}$ ) have been computed in a series of Monte Carlo experiments. Applying (7) to data generated by (5) with $b=0$ gives approximate distributions of relevant statistics under the null hypothesis in (A). Similarly, applying (7) to data generated by (5) with non-zero values of $b$ gives distributions of the statistics in (B) under the null hypothesis. In our experiments the parameter $b$ has been chosen in such a way that the largest one corresponds to $b=1 / \sqrt{T}$ and the subsequent $b$ 's are proportionally smaller; $b$ is defined as $b=d / \sqrt{T}$, where $d=1,0.5,0.25$ and 0 . For $d=0$, the data generating process correspond to the null hypothesis in (A), otherwise it is that of (B). Value of $T_{B}$, that is, time indicator of a break, see (3) and/or (4), has been set at 0.85 T. This corresponds to the empirical example used in this paper (see further in Section 3). For each set of parameters and each sample size $(T=1,000,1,500,2,500), 100,000$ replications were made. It turned out that sample size do not affect the results markedly.

Table 1 presents the relevant percentiles obtained in each experiment. The way of using this table is the following. To test the set of (A) hypotheses use values given in column headed $d=0$. For testing the set of (B) hypotheses, it is necessary to compute $\delta=\hat{b} \sqrt{T}$. Next, choose a column headed by $d$ to which $\delta$ could be rounded and use its percentiles as the critical values.

\section{Empirical Results}

In the empirical analysis we use daily (session-to session) data on prices and returns on 66 stock markets worldwide for the period from the $23^{\text {rd }}$ of March 1992 until the $6^{\text {th }}$ of December 2001. The length of the series varies from 1100 to 2534. It is assumed here that the possible breakpoint in the series, that is $T_{B}$ in (3) and/or (4), is unknown. As the criteria for selection of the break date we use Zivot and Andrews (1992) criterion, according to which the break is in the period where the linear unit root $t$-statistic is the smallest. Other criteria considered by Vogelsang and Perron (1998) were also used, giving 
Table 1: Critical values for the encompassing test

\begin{tabular}{|c|c|c|c|c|c|c|}
\hline \multirow[t]{3}{*}{ Test } & percent. & & $d=0$ & $d=0.25$ & $d=0.50$ & $d=1$ \\
\hline & $1 \%$ & & -3.45 & -3.04 & -2.85 & -2.56 \\
\hline & $5 \%$ & & -2.75 & -2.38 & -2.12 & -1.61 \\
\hline \multirow{6}{*}{$t(b)$} & $10 \%$ & & -2.40 & -1.98 & -1.69 & -1.05 \\
\hline & $90 \%$ & & 2.40 & 3.11 & 3.96 & 5.96 \\
\hline & $95 \%$ & & 2.75 & 3.75 & 5.41 & 9.09 \\
\hline & $99 \%$ & & 3.45 & 5.62 & 10.37 & 16.19 \\
\hline & $1 \%$ & as & in $N(0,1)$ & -2.95 & -3.38 & -3.87 \\
\hline & $5 \%$ & as & in $N(0,1)$ & -2.04 & -2.11 & -2.93 \\
\hline \multirow[t]{5}{*}{$t(\gamma)$} & $10 \%$ & as & in $N(0.1)$ & -1.55 & -1.60 & -2.25 \\
\hline & $90 \%$ & as & in $N(0,1)$ & 1.83 & 2.19 & 2.76 \\
\hline & $95 \%$ & as & in $N(0,1)$ & 2.40 & 2.82 & 3.57 \\
\hline & $99 \%$ & as & in $N(0,1)$ & 3.54 & 4.04 & 4.96 \\
\hline & $90 \%$ & & 4.47 & $\mathrm{~N} / \mathrm{A}$ & N/A & $\mathrm{N} / \mathrm{A}$ \\
\hline \multirow[t]{2}{*}{$F(b, \gamma)$} & $95 \%$ & & 5.42 & $\mathrm{~N} / \mathrm{A}$ & N/A & $\mathrm{N} / \mathrm{A}$ \\
\hline & $99 \%$ & & 7.53 & N/A & N/A & $\mathrm{N} / \mathrm{A}$ \\
\hline
\end{tabular}

similar results.

It can be concluded that the $U R B$ process without a break encompasses the $U R D$ process with a break if for (7) the $F$-statistic is significant, $b$ is significant under (A) and (B) and $\gamma$ is not significant under (B). Analogously, the $U R D$ process with a deterministic break encompasses the $U R B$ process without a break if, in addition to the $F$-statistic being significant in (A), $\gamma$ is significant under (B) and $b$ is not significant under (C).

The detailed results of the encompassing exercise are presented at Table 2 at the Appendix $\mathrm{B}$. They indicate that the $U R B$-no break test shows, on the balance, some advantage over the $U R D$-break approach. In 26 cases out of 66 the result is such that the $t$-statistic for $\hat{b}$ under the set of hypotheses (C) remains significant while the corresponding statistic for $\hat{\gamma}$ loses its significance under the set of hypotheses (B). Since in all these cases the $U R D$ model, while tested separately, appears to be significant, it might be concluded that a true bilinear unit root process might frequently be mistaken for the deterministic unit root process with a structural break. In 6 cases, however, the $U R D$ model seems to encompass the $U R B$ model. This includes the important USA market, for which the deterministic unit root model with a structural break appears to be superior. Among 6 countries for which the $U R D$ model encompasses $U R B$, there is only one, Jordania, which is not within the $G 25$ group of the 25 richest (in terms of per capita real GDP) countries. Additionally we have found that only 6 countries belonging to the $G 25$ group are among the 26 for which the $U R B$ model encompasses $U R D$. Hence, it seems that unit root bilinearity is more typical for the emerging and developing markets rather than for the mature markets.

Under (B), a relatively large number of insignificant results suggest that the problem of identification of underlying stochastic patterns for financial time series is far from being solved. True dynamics of these series is presumably more complicated and involves a mixture of a stochastic unit root process with that of a deterministic break. This, however, 
requires further investigation.

\section{Conclusions}

Stochastic unit root modelling and, in particular, the bilinear unit root approach presented here, offers an attractive alternative to the traditional (deterministic) unit root analysis. The concept of the bilinear unit roots can substantially enrich the analysis traditionally conducted within the deterministic unit root framework. More specifically, a possible speculative bubble interpretation of the $U R B$ model and the computationally simple nature of its tests create an interesting tool for the analysis of ups and downs on financial markets. So far, speculative bubbles have been regarded as difficult to treat on the grounds of unit root econometrics (see e.g. Evans, 1991). Results presented here also reveal that a substantial number of the empirical financial time series exhibits unit root bilinearity. Testing for this particular form of the stochastic unit root is feasible and can be done without the need for developing specialised software. Finally the URB process, being lowly parameterised, does not require specific assumptions or additional tests regarding the nature or timing of the structural breaks.

\section{Appendix A. Proofs of Lemma and Theorem}

Proof of the Lemma. Consider the data generating process $(D G P)$ given by equation:

$$
y_{t}=y_{t-1}+\varepsilon_{t}, \varepsilon_{t} \sim \operatorname{IID}\left(0, \sigma^{2}\right), y_{0}=0, t=1,2, \ldots, T
$$

For the parameters of the equation of interest (7) the usual OLS estimator is given as:

$$
[\hat{c}, \hat{b}, \hat{\gamma}]^{\prime}=\left(X^{\prime} X\right)^{-1} X^{\prime} Y
$$

where:

$$
X=\left[\begin{array}{ccc}
1 & y_{1} \Delta y_{1} & 0 \\
1 & y_{1} \Delta y_{1} & 0 \\
\cdots & \cdots & \cdots \\
1 & y_{T_{B}-2} \Delta y_{T_{B}-2} & 0 \\
1 & y_{T_{B}-1} \Delta y_{T_{B}-1} & 1 \\
\cdots & \ldots & \cdots \\
1 & y_{T-1} \Delta y_{T-1} & 1
\end{array}\right], \text { and } Y=\left[\begin{array}{c}
\Delta y_{2} \\
\Delta y_{3} \\
\cdots \\
\Delta y_{T}
\end{array}\right]
$$

and $T_{B}$ is a moment of a possible break. To obtain the asymptotic of matrices $X^{\prime} X$ and $X^{\prime} Y$ (with $X$ and $Y$ defined by (13)) under the DGP of (11) and the null hypothesis of $b=\gamma=0$, let us apply the Donsker 's theorem and some results of Charemza et al. (2005), namely of the Statement, section 1 and Lemma C, sections (3) and (4), which 
give:

$$
\begin{aligned}
& T^{-1}\left(X X^{\prime}\right)_{11} \Rightarrow 1, T^{-1}\left(X X^{\prime}\right)_{12} \Rightarrow \sigma^{2}\left(\int_{0}^{1} W_{1}(t) d W_{1}(t)+1\right), \\
& T^{-1}\left(X X^{\prime}\right)_{13} \Rightarrow 1-\lambda, T^{-2}\left(X X^{\prime}\right)_{22} \Rightarrow \sigma^{4} \int_{0}^{1} W_{1}^{2}(t) d t, \\
& T^{-1}\left(X X^{\prime}\right)_{23} \Rightarrow(1-\lambda) \sigma^{2}\left(\int_{0}^{1} W_{1}(t) d W_{1}(t)+1\right), T^{-1}\left(X X^{\prime}\right)_{31} \Rightarrow 1-\lambda,
\end{aligned}
$$

and

$$
\begin{aligned}
& T^{-1 / 2}\left(X^{\prime} Y\right)_{11} \Rightarrow \sigma W(1), T^{-1}\left(X^{\prime} Y\right)_{21} \Rightarrow \sigma^{3} \int_{0}^{1} W_{1}(t) d W_{2}(t), \\
& T^{-1 / 2}\left(X^{\prime} Y\right)_{31} \Rightarrow(1-\sqrt{\lambda}) \sigma W(1),
\end{aligned}
$$

where $\left(X X^{\prime}\right)_{i j}$ and $\left(X^{\prime} Y\right)_{i 1}(i, j=1,2,3)$ are corresponding elements of matrices $X^{\prime} X$ and $X^{\prime} Y, \Rightarrow$ denotes weak convergence, $W_{1}, W_{2}$ are two independent Wiener processes on $[0,1]$ and $\lambda$ is defined by (9).

Combination of (12), (14) and (15) after some usual algebra complete the proof of the Lemma.

Proof of the Theorem. The test statistics for $b=\gamma=0$ in (7) has a form of the F-test:

$$
F=\frac{\left(R S S_{(R)}-R S S_{(U R)}\right) / 2}{R S S_{(U R)} /(T-4)},
$$

where $R S S_{(R)}$ is sum of squared $O L S$-residuals from the restricted regression, that is:

$$
\Delta y_{t}=c+w_{t}, \quad(t=2,3, \ldots, T),
$$

and $R S S_{(U R)}$ is sum of squared $O L S$-residuals from the unrestricted regression, that is from (7). For the restricted model (17) sum of squared $O L S$-residuals are:

$$
R S S_{(R)}=\sum_{t=2}^{T} \hat{w}_{t}^{2}=\sum_{t=2}^{T}\left(\Delta y_{t}\right)^{2}-T\left(\frac{1}{T} \sum_{t=2}^{T} \Delta y_{t}\right)^{2},
$$

and, under the DGP of (11) and the null hypothesis of $b=\gamma=0$, we get:

$$
T \sum_{t=2}^{T}\left(\Delta y_{t}\right)^{2}=T \sum_{t=2}^{T} \varepsilon_{t}^{2} \Rightarrow \sigma^{2}
$$

and:

$$
T\left(\frac{1}{T} \sum_{t=2}^{T} \Delta y_{t}\right)^{2}=\left(\frac{1}{\sqrt{T}} \sum_{t=2}^{T} \varepsilon_{t}\right)^{2} \Rightarrow\left[\sigma W_{1}(1)\right]^{2} .
$$

Sum of squared $O L S$-residuals from the unrestricted regression of (7) with the use of (12) and (13) may be decomposed as:

$$
\begin{aligned}
R S S_{(U R)}=\sum_{t=2}^{T} e_{t}^{2}= & \sum_{t=2}^{T}\left(\Delta y_{t}\right)^{2}+T \hat{c}+\hat{b}^{2} \sum_{t=2}^{T}\left(y_{t-1} \Delta y_{t-1}\right)^{2}-2 \hat{c} \sum_{t=2}^{T} \Delta y_{t-1} \\
& -2 \hat{b} \sum_{t=2}^{T} y_{t-1} \Delta y_{t-1} \Delta y_{t}+2 \hat{b} \hat{c} \sum_{t=2}^{T} y_{t-1} \Delta y_{t-1}-2 \hat{\gamma} \sum_{t=T_{B}}^{T} \Delta y_{t-1} \\
& +2 \hat{\gamma} \hat{c}\left(T-T_{B}\right)+2 \hat{\gamma} \hat{b} \sum_{t=T_{B}}^{T} y_{t-1} \Delta y_{t-1}+\hat{\gamma}^{2}\left(T-T_{B}\right) .
\end{aligned}
$$


From, (18), (19) and (20) we immediately obtain that, under the null hypothesis and as $T \rightarrow \infty$, the denominator in (16) will converge to $\sigma^{2}$, that is:

$$
(T-4)^{-1} R S S_{(U R)}=(T-4)^{-1}\left(\sum_{t=2}^{T}\left(\varepsilon_{t}\right)^{2}-T\left(\frac{1}{T} \sum_{t=2}^{T} \varepsilon_{t}\right)^{2}\right) \Rightarrow \sigma^{2} .
$$

For the nominator in (16), applying the Lemma for decomposition (21), we obtain:

$$
R S S_{(R)}-R S S_{(U R)} \Rightarrow \sigma^{2} \frac{\left(\int_{0}^{1} W_{1}(t) d W_{2}(t)\right)^{2}}{\int_{0}^{1} W_{1}^{2}(t) d t}+\sigma^{2} \frac{(1-\sqrt{\lambda})(4-\lambda)}{\lambda(1+\sqrt{\lambda})} W_{1}^{2}(1) .
$$

Combination of (16), (22) and (23) complete the proof the of Theorem.

\section{Appendix B. Empirical Results}

Table 2 presents the empirical results of the encompassing test exercise. After names of particular countries, the $F$-statistics for testing the joint significance of the parameters $b$ and $\gamma$ under the set of hypotheses (A) in (7) are given. Their significance is indicated in the third column as follows: ' 0 ' means no significance, ' + ', ' ++ ' and ' +++ ' means that the statistic is significant respectively at $0.10,0.05$ and 0.01 level of significance. Column 4 gives the estimates of the $t$-statistics for $b$. The next two columns show their significance in (A) and (B). The last three columns show the values of Student- $t$ statistics for $\gamma$ in (A) and (B) respectively. The cases where the $t$-statistic for $b$ under the set of hypotheses (B) remains significant while the corresponding statistic for $\gamma$ loses its significance are in italics. The codes for countries which belong to the richest (in terms of per capita real $G D P$, the so called $G 25$ group) are marked by *.The 6 cases, for which the URD model seems to encompass the $U R B$ model are marked by boldfacing.

\section{References}

W.W. Charemza, M. Lifshits, and S. Makarova. Conditional testing for unit-root bilinearity in financial time series: some theoretical and empirical results. Journal of Economic Dynamics and Control, 29:63-96, 2005.

G.W. Evans. Pitfalls in testing for explosive bubbles in asset pricing. American Economic Review, 81:922-930, 1991.

C.W.J. Granger and A.P. Andersen. An introduction to bilinear time series models. Vandenhoeck and Ruprecht, Göttingen, 1978.

C.W.J. Granger and N.R. Swanson. An introduction to stochastic unit-root processes. Journal of Econometrics, 80:35-62, 1997. 
D.I. Harvey and T.C. Mills. Tests for stationarity in series with endogenously determined structural change. In European Meeting of the Econometric Society, Venice, 2002.

T-H. Kim, S.J. Leybourne, and P. Newbold. Spurious rejection by perron test in the presence of a break. Oxford Bulletin of Economics and Statistics, 62:433-444, 2000.

S.J. Leybourne, B.P.M. McCabe, and A.R. Tremayne. Can economic time series be differenced to stationarity? Journal of Business and Economic Statistics, 14:435-446, 2000.

M. Lifshits. Behaviour of statistical aggregates under small bilinearity. St. Petersburg State University, mimeo, 2002.

B.P.M. McCabe and A.R. Tremayne. Testing a time series for difference stationarity. The Annals of Statistics, 23:1015-1028, 1995.

S. Ng and P. Perron. Lag length selection and the construction of unit root tests with good size and power. Econometrica, 69:1519-1554, 2001.

P. Perron. The great crash, the oil price shock and the unit root hypothesis. Econometrica, 57:1361-1401, 1989.

P. Perron. Testing for a unit root in a time series with a changing mean. Journal of Business and Economic Statistics, 8:153-162, 1990.

P. Perron. Further evidence on breaking trend functions in macroeconomic variables. Journal of Econometrics, 80:355-385, 1997.

R. Sollis, S.J. Leybourne, and P. Newbold. Stochastic unit roots modelling of stock price indices. Applied Financial Economics, 10:311-315, 2000.

T.J. Vogelsang and P. Perron. Additional tests for a unit root allowing for a break in the trend function at an unknown time. International Economic Review, 39:1073-1100, 1998.

E. Zivot and D.W.K. Andrews. Further evidence of the great crash, the oil shock and the unit root hypothesis. Journal of Business and Economic Statistics, 10:251-270, 1992.

Corresponding author's address:

Prof. Wojciech W. Charemza

Department of Economics

The University of Leicester

University Road

Leicester, England, LEI 7RH

Tel. +44 1162522899

Fax +44 1162522908

E-mail: wch@le.ac.uk

http://www.le.ac.uk/economics/wch/ 
Table 2: Results of encompassing test

\begin{tabular}{|c|c|c|c|c|c|c|c|c|}
\hline \multirow{3}{*}{ Code } & \multicolumn{3}{|c|}{ Bilinearity stat. } & \multicolumn{5}{|c|}{ Break statistic } \\
\hline & \multirow{2}{*}{$F(b, \gamma)$} & \multirow{2}{*}{$\begin{array}{r}\text { Snfce } \\
(A)\end{array}$} & \multirow[t]{2}{*}{$t(b)$} & \multicolumn{2}{|c|}{ Snfce } & \multirow[t]{2}{*}{$t(\gamma)$} & \multicolumn{2}{|c|}{ Snfce } \\
\hline & & & & (A) & (B) & & (A) & (B) \\
\hline ARGENTINA & 33.59 & +++ & 5.62 & +++ & 0 & -1.43 & 0 & 0 \\
\hline AUSTRALIA* & 7.67 & +++ & -2.72 & +++ & + & -0.54 & 0 & 0 \\
\hline AUSTRIA* & 5.45 & ++ & -2.32 & +++ & 0 & -0.23 & 0 & 0 \\
\hline BANGLADESH & 15.57 & +++ & 3.89 & +++ & 0 & 0.65 & 0 & 0 \\
\hline BELGIUM* & 102.76 & +++ & 10.01 & +++ & ++ & -1.63 & 0 & 0 \\
\hline BRAZIL & 21.36 & +++ & 4.60 & +++ & 0 & -0.40 & + & 0 \\
\hline CANADA* & 8.44 & +++ & -2.60 & +++ & + & -1.30 & 0 & 0 \\
\hline CHILE & 155.72 & +++ & 12.48 & +++ & ++ & -0.13 & 0 & 0 \\
\hline CHINA & 2.58 & 0 & 1.23 & +++ & 0 & -1.04 & 0 & 0 \\
\hline COLUMBIA & 6.39 & ++ & -2.53 & +++ & + & 0.11 & 0 & 0 \\
\hline CROATIA & 4.33 & 0 & 2.04 & +++ & 0 & 0.42 & 0 & 0 \\
\hline CYPRUS* & 34.76 & +++ & 5.83 & +++ & 0 & -0.85 & 0 & 0 \\
\hline CZECH REPUBLIC & 43.18 & +++ & 6.57 & +++ & + & -0.06 & 0 & 0 \\
\hline DENMARK* & 8.83 & +++ & -2.21 & +++ & 0 & -1.99 & ++ & ++ \\
\hline EGYPT & 10.71 & +++ & 2.87 & ++ & 0 & -1.57 & 0 & 0 \\
\hline ESTONIA & 1.33 & 0 & -1.13 & +++ & 0 & 0.23 & 0 & 0 \\
\hline FINLAND* & 13.88 & +++ & -3.46 & +++ & +++ & -1.38 & + & 0 \\
\hline FRANCE* & 10.34 & +++ & 2.67 & + & 0 & -1.79 & 0 & ++ \\
\hline GERMANY* & 10.19 & +++ & 2.82 & ++ & 0 & -1.50 & 0 & 0 \\
\hline GREECE & 59.42 & +++ & 7.62 & +++ & + & -1.17 & 0 & 0 \\
\hline HONG KONG* & 6.96 & ++ & 2.48 & + & 0 & -0.91 & 0 & 0 \\
\hline HUNGARY & 18.39 & +++ & -4.25 & +++ & +++ & -0.58 & 0 & 0 \\
\hline ICELAND* & 13.85 & +++ & 3.58 & +++ & 0 & -1.01 & 0 & 0 \\
\hline INDIA & 44.84 & +++ & 6.68 & +++ & + & -0.43 & 0 & 0 \\
\hline INDONESIA & 46.91 & +++ & 6.79 & +++ & + & -0.90 & 0 & 0 \\
\hline IRELAND* & 7.48 & ++ & -2.62 & +++ & + & -0.79 & 0 & 0 \\
\hline ISRAEL* & 3.73 & 0 & 1.19 & +++ & 0 & -1.52 & 0 & 0 \\
\hline ITALY* & 5.58 & ++ & -1.76 & +++ & 0 & -1.57 & + & 0 \\
\hline JAPAN* & 5.50 & ++ & 2.09 & +++ & 0 & -1.06 & 0 & 0 \\
\hline JORDANIA & 15.81 & +++ & 3.47 & +++ & 0 & 1.93 & 0 & ++ \\
\hline KOREA, REP OF & 7.86 & +++ & 2.76 & ++ & 0 & 0.49 & 0 & 0 \\
\hline LATVIA & 17.39 & +++ & 4.17 & +++ & 0 & 0.18 & 0 & 0 \\
\hline LEBANON & 46.57 & +++ & 6.78 & +++ & + & -0.74 & 0 & 0 \\
\hline LITHUANIA & 45.72 & +++ & 6.76 & +++ & + & -0.17 & 0 & 0 \\
\hline LUXEMBOURG* & 63.35 & +++ & 7.42 & +++ & + & -2.87 & + & +++ \\
\hline MALAYSIA & 21.76 & +++ & 4.66 & +++ & 0 & -0.22 & 0 & 0 \\
\hline MAURITIUS & 11.40 & +++ & 3.05 & ++ & 0 & -1.44 & 0 & 0 \\
\hline MEXICO & 72.29 & +++ & 8.48 & +++ & + & -0.59 & 0 & 0 \\
\hline MOROCCO & 68.73 & +++ & 8.24 & +++ & + & -0.90 & 0 & 0 \\
\hline NETHERLANDS* & 10.18 & +++ & 2.01 & +++ & 0 & -2.48 & + & +++ \\
\hline NIGERIA & 0.27 & 0 & -0.52 & +++ & 0 & 0.07 & 0 & 0 \\
\hline NORWAY* & 5.91 & ++ & -2.00 & +++ & 0 & -1.37 & + & 0 \\
\hline
\end{tabular}


Table 2 (cont.): Results of encompassing test

\begin{tabular}{|l|r|r|r|r|r|r|r|r|}
\hline & \multicolumn{4}{|c|}{ Bilinearity stat. } & \multicolumn{4}{c|}{ Break statistic } \\
\hline Code & $F(b, \gamma)$ & Snfce & $t(b)$ & \multicolumn{2}{|c|}{ Snfce } & $t(\gamma)$ & \multicolumn{2}{|c|}{ Snfce } \\
\hline & & $(\mathbf{A})$ & & $\mathbf{( A )}$ & $\mathbf{( B )}$ & & $\mathbf{( A )}$ & $\mathbf{( B )}$ \\
\hline NEW ZEALAND* & 0.03 & 0 & 0.17 & +++ & 0 & -0.02 & 0 & 0 \\
\hline PAKISTAN & 18.89 & +++ & 4.34 & +++ & 0 & -0.27 & 0 & 0 \\
\hline PERU & 85.86 & +++ & 9.26 & +++ & ++ & -0.45 & 0 & 0 \\
\hline PHILIPPINES & 90.71 & +++ & 9.48 & +++ & ++ & -0.88 & 0 & 0 \\
\hline POLAND & 5.33 & + & -2.30 & +++ & 0 & -0.20 & 0 & 0 \\
\hline PORTUGAL & 9.12 & +++ & -2.61 & +++ & + & -1.51 & + & 0 \\
\hline ROMANIA & 2.65 & 0 & -1.50 & +++ & 0 & 0.64 & 0 & 0 \\
\hline RUSSIA & 3.32 & 0 & -1.82 & +++ & 0 & 0.01 & 0 & 0 \\
\hline SOUTH AFRICA & 49.80 & +++ & 7.06 & +++ & + & 0.00 & 0 & 0 \\
\hline SINGAPORE* & 22.89 & +++ & 4.76 & +++ & 0 & -0.44 & 0 & 0 \\
\hline SLOVAKIA & 9.14 & +++ & -2.83 & +++ & ++ & 1.08 & 0 & 0 \\
\hline SLOVENIA & 234.24 & +++ & 15.29 & +++ & ++ & 0.71 & 0 & 0 \\
\hline SPAIN* & 6.06 & ++ & -2.26 & +++ & 0 & -0.97 & 0 & 0 \\
\hline SRI LANKA & 273.56 & +++ & 16.50 & +++ & +++ & 1.14 & 0 & 0 \\
\hline SWITZERLAND* & 16.14 & +++ & 3.45 & +++ & 0 & -2.05 & 0 & ++ \\
\hline SWEDEN* & 18.65 & +++ & -4.15 & +++ & +++ & -1.18 & 0 & 0 \\
\hline TAIWAN CHINA & 0.75 & 0 & 0.81 & +++ & 0 & -0.32 & 0 & 0 \\
\hline THAILAND & 39.07 & +++ & 6.25 & +++ & + & 0.00 & 0 & 0 \\
\hline TURKEY & 20.93 & +++ & -4.51 & +++ & +++ & -0.74 & 0 & 0 \\
\hline U. K. $*$ & 18.65 & +++ & 4.02 & +++ & 0 & -1.58 & 0 & 0 \\
\hline UNITED STATES* & 5.46 & ++ & 1.43 & +++ & 0 & -1.85 & + & ++ \\
\hline VENEZUELA & 10.84 & +++ & 3.14 & ++ & 0 & -1.00 & 0 & 0 \\
\hline ZIMBABWE & 283.14 & +++ & 16.82 & +++ & +++ & 0.60 & 0 & 0 \\
\hline
\end{tabular}

